\title{
Potential applications of nanotechnology in thermochemical conversion of microalgal biomass
}

\begin{abstract}
The rapid decrease in fossil reserves has significantly increased the demand of renewable and sustainable energy fuel resources. Fluctuating fuel prices and significant greenhouse gas (GHG) emission levels have been key impediments associated with the production and utilization of nonrenewable fossil fuels. This has resulted in escalating interests to develop new and improve inexpensive carbon neutral energy technologies to meet future demands. Various process options to produce a variety of biofuels including biodiesel, bioethanol, biohydrogen, bio-oil, and biogas have been explored as an alternative to fossil fuels. The renewable, biodegradable, and nontoxic nature of biofuels make them appealing as alternative fuels. Biofuels can be produced from various renewable resources. Among these renewable resources, algae appear to be promising in delivering sustainable energy options. Algae have a high carbon dioxide (CO2) capturing efficiency, rapid growth rate, high biomass productivity, and the ability to grow in non-potable water. For algal biomass, the two main conversion pathways used to produce biofuel include biochemical and thermochemical conversions. Algal biofuel production is, however, challenged with process scalability for high conversion rates and high energy demands for biomass harvesting. This affects the viable achievement of industrial-scale bioprocess conversion under optimum economy. Although algal biofuels have the potential to provide a sustainable fuel for future, active research aimed at improving upstream and downstream technologies is critical. New technologies and improved systems focused on photobioreactor design, cultivation optimization, culture dewatering, and biofuel production are required to minimize the drawbacks associated with existing methods. Nanotechnology has the potential to address some of the upstream and downstream challenges associated with the development of algal biofuels. It can be applied to improve system design, cultivation, dewatering, biomass characterization, and biofuel conversion. This chapter discusses thermochemical conversion of microalgal biomass with recent advances in the application of nanotechnology to enhance the development of biofuels from algae. Nanotechnology has proven to improve the performance of existing technologies used in thermochemical treatment and conversion of biomass. The different bioprocess aspects, such as reactor design and operation, analytical techniques, and experimental validation of kinetic studies, to provide insights into the application of nanotechnology for enhanced algal biofuel production are addressed.
\end{abstract}

Keyword: Microalgae; Gasification; Nanotechnology; Thermogravimetric analysis; Thermochemical conversion 